\title{
Clinical efficacy of intermittent magnetic pressure therapy for ear keloid treatment after excision
}

\author{
Dongkeun Jun, \\ Donghyeok Shin, \\ Hyungon Choi, \\ Myungchul Lee \\ Department of Plastic and \\ Reconstructive Surgery, Konkuk \\ University School of Medicine, Seoul, \\ Korea
}

\begin{abstract}
Background: Keloids are benign fibro-proliferative lesion, related to excessive inflammatory reactions in certain anatomical areas, including the auricles. Their specific etiology remains unclear; nonetheless they exhibit tumor-like characteristics of significant recurrence and cause emotional distress, even with various treatment strategies. We applied intermittent magnetic pressure therapy on ear keloids in combination with surgical excision, and present its effectiveness herein.

Methods: Ear keloid patients were treated with surgical excision followed by magnetic pressure therapy. The keloid tissues underwent excision and keloid marginal flaps were utilized for wound closure. Intermittent magnetic pressure therapy was applied 2 weeks after the surgical procedure. The pressure therapy consisted of a 3-hour application and 2-hour resting protocol (9 hr/day), and lasted for 6 months. The results were analyzed 6 months after the therapeutic procedures, using the scar assessment scale.

Results: Twenty-two ear keloids from 20 patients were finally reviewed. Among the keloids that completed the therapeutic course, 20 ear keloids out of 22 in total (90.9\%) were successfully eradicated. Two patients (2 keloids) exhibited slight under-correction. Postoperative complications such as wound dehiscence or surgical site infection were not noted. The scar assessment scale demonstrated a significant improvement in each index. The intermittent pressure therapy led to patient compliance, and avoided pressure-related pain and discomfort.

Conclusion: Excision followed by intermittent pressure application using a magnet successfully reduced the burden of fibro-proliferative keloids, and had good patient compliance. The role of intermittent pressure application and resting should be studied with regard to keloid tissue remodeling.
\end{abstract}

Keywords: Keloid / Magnet / Pressure / Therapy

\section{INTRODUCTION}

Keloids are fibro-proliferative accumulation of extracellular matrices, especially collagen fibers, which are induced in the circumstances of exaggerated expression of cytokine and growth factors. They are one of the most common complica-

Correspondence: Myungchul Lee

Department of Plastic and Reconstructive Surgery, Konkuk University School of

Medicine, 120-1 Neungdong-ro, Gwangjin-gu, Seoul 05030, Korea

E-mail: mcle1999@gmail.com

Received September 5, 2019 / Revised October 19, 2019 / Accepted October 21, 2019 tions in patients who have had mechanical trauma, undergone surgery, or have an inflammation history in the auricular area. Although the specific etiology of keloids remains unclear, they seem to develop following traumatic dermal injuries in patients with genetic predispositions or past keloid histories [1].

Because keloid scars show benign skin tumor-like properties, such as a continuous growth tendency, they are excised with primary closure and treated with multimodal adjuvant therapies, which include intralesional steroid injections, radiation treatment, pressure therapy, and cryotherapy [2]. These affect 
the healing process to be finalized adequately until the epithelialization of the wound [3]. However, even with the multimodal management procedures, a complete resolution of the recurrence issue seems unaccomplished [4].

For the keloid tissue suppressing effect, continuous applying of the pressure devices has been introduced and recommended in most studies. Park et al. [5] reported the efficacy of magnetic disks for earlobe keloids which is applied to the patients for 12 hours per day for 6 months following surgical excisions, whereas Tanaydin et al. [6] suggested the use of pressure clips for at least 12 hours per day for 6 to 18 months as an adjuvant treatment of the ear keloids following surgical excisions. However, in various clinical trials, there have been no definite application protocols or discussions on how to apply the pressure generating devices specifically.

An intermittent application concept of mechanical forces has been introduced from other wound treatment methods. The effectiveness of the intermittent application of the mechanical pressure or elastic traction was reported in former studies [7-9]. We tried to adopt this concept into keloid adjuvant therapy, so that we could accomplish to set up a patient-convenient combinational modality after surgical excision treatments.

\section{METHODS}

Patients with ear keloids were reviewed in this retrospective study. The patients were treated with surgical excision followed by magnetic pressure therapy over a period of 3 years, from May 2015 to April 2018, at Konkuk University Medical Center. The study protocol conformed to the ethical guidelines of the Declaration of Helsinki, as reflected in the approval by the Konkuk University School of Medicine human research review committee.

Patient data were collected and reviewed in accordance with the following inclusion criteria: (1) primary and secondary keloids that had developed on the auricular area caused by ear trauma or piercings; (2) keloids that protruded and extended beyond the margin of the initial injury lesion; (3) patients who underwent surgical excision with primary closure; and (4) patients who did not undergo additional ear piercing or surgical procedures during the follow-up period.

Patients were excluded when their follow-up data were not available. All studied patients complied with our treatment protocol, and consented to the final follow-up after 6 months since the completion of management.

\section{Surgical techniques}

Surgical procedures were performed under local anesthesia. We performed near-complete excision, 1-2 mm within the keloid edge. The keloid marginal flap, which consisted of the epidermis and the thin dermis overlying the keloid, was raised from the fibrous keloid core. The flap was utilized for wound closure withounsion. Bleeding was controlled using bipolar coagulation. We closed wounds with appropriate approximation using Prolene 5-0 and 6-0 interrupted sutures (Fig. 1). Compressive dressing was applied to prevent hematoma. The stitches were removed on postoperative days 7 to 14 . Pain was controlled with the administration of nonsteroidal anti-inflammatory medication.

\section{Intermittent magnetic pressure therapy protocol}

Intermittent magnetic pressure therapy was initiated 2 weeks after the surgical procedure. In case of delayed wound healing, due to an unstable wound margin and serous discharge, the intermittent pressure therapy was considered 3 weeks postopera-

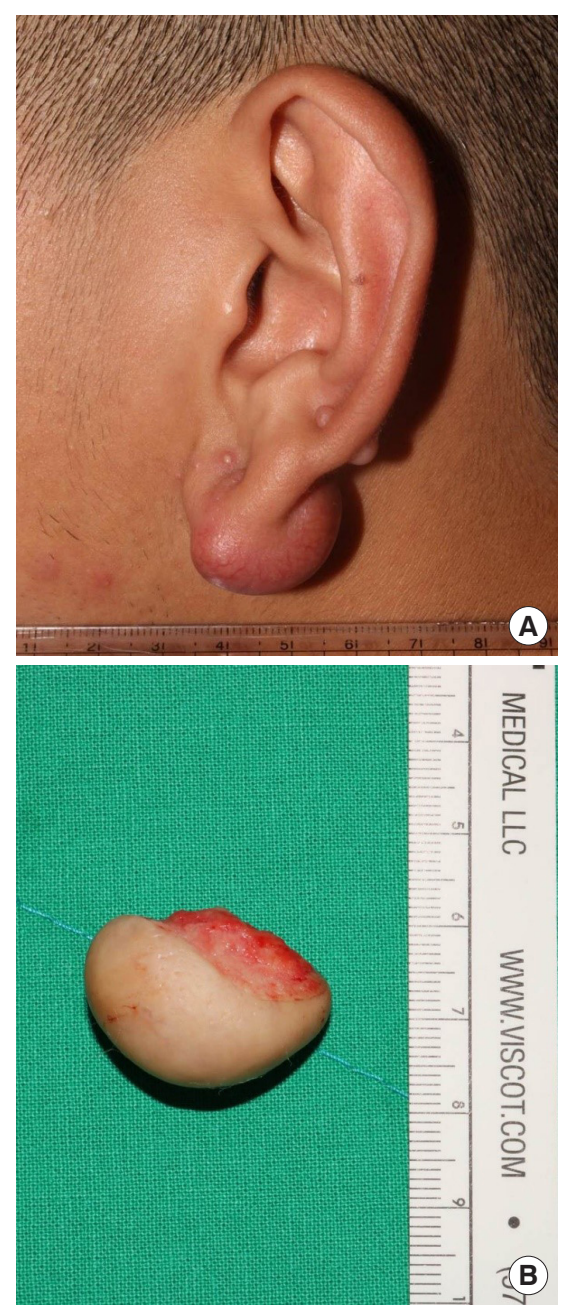

Fig. 1. (A) A 20-year-old male patient presented a pedunculatedpattern keloid on the left ear lobule. (B) Excised keloid was composed of whitish connective tissue, measured $1.5 \times 1.5 \mathrm{~cm}$. 
tively. Patients were instructed to use the magnets for approximately 9 hours per day for 6 months until the completion of the therapeutic procedures. During the adjuvant pressure therapy, patients were observed frequently for checking recurrence and for wound evaluation. Our protocol included three cycles of magnet application per day, with intermittent application throughout the day. Each cycle was composed of 3 hours of magnet application followed by 2 hours of relief. The pressure therapy protocol was designed to avoid patient discomfort, achieving compressive effects. Three alternative magnets were utilized and a lighter magnet with less pull strength was applied when patients reported discomfort or pain (Fig. 2). The pressure between pair of magnets was measured using a digital manometer (Rupse HT-1895; Rupse, Hong Kong, People’s Republic of China). The three magnets demonstrated values in regular sequence (magnet diameter $8 \mathrm{~mm}, 24 \mathrm{kPa}$; diameter $10 \mathrm{~mm}, 42$ $\mathrm{kPa}$; diameter $12 \mathrm{~mm}, 60 \mathrm{kPa}$ ).

\section{Outcome measurement}

With regard to the comparative analysis between pre- and postoperative states, the scar assessment scale was adopted from previous studies on keloid scar treatments [10]. The scar assessment scale was assigned by three different plastic surgeons and the median value was utilized for analysis. Patients' satisfaction was evaluated on the basis of symptom relief during the postoperative follow-up visits. The postoperative assessment was performed at the final follow-up after 6 months, since the completion of intermittent pressure therapy.

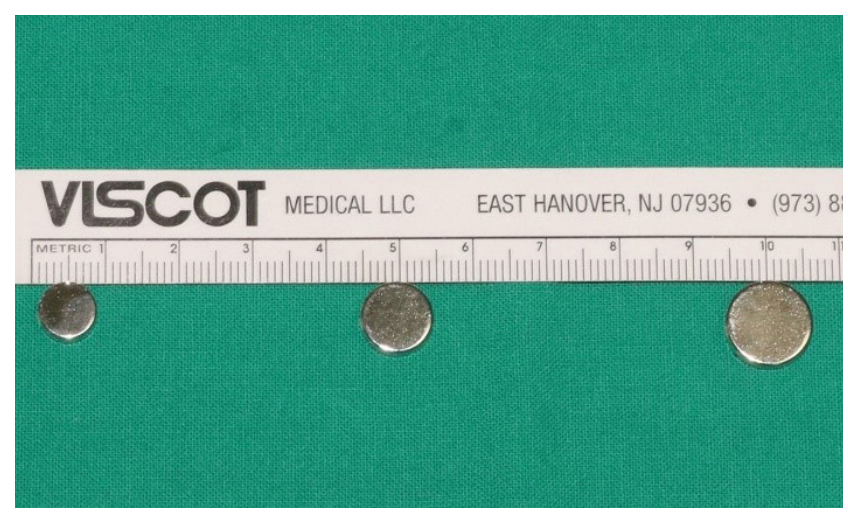

Fig. 2. Intermittent pressure therapy protocol included 3 cycles of magnet application per day. Each cycle consisted of 3 hours of magnet application followed by 2 hours of relief $(9 \mathrm{hr} /$ day). The protocol was introduced to avoid patient discomfort, allowing compressive effects. Three alternative magnets were used and a lighter magnet with less pull strength was applied when patients notified discomfort or pain. With regard to the pressure measurement, three magnets presented values in regular sequence (magnet diameter $8 \mathrm{~mm}$, $24 \mathrm{kPa}$; diameter $10 \mathrm{~mm}, 42 \mathrm{kPa}$; diameter $12 \mathrm{~mm}, 60 \mathrm{kPa}$ ).

\section{Statistical analysis}

For each scale index, values at pre- and post-treatments were compared using a two-tailed paired $t$-test. All data were expressed as mean \pm standard deviation. Data analyses were performed with standard software SPSS for Windows version 22.0 (IBM Corp., Armonk, NY, USA). Statistical significance was assumed if $p$-value was less than 0.05 .

\section{RESULTS}

Twenty patients with ear keloids were finally included in the study, and a total of 22 ear keloid cases were reviewed. The average patient age was 22 years (range, $15-40$ years). The average diameter of keloids was $1.7 \pm 0.8 \mathrm{~cm}$, ranging from 0.9 to 2.8 $\mathrm{cm}$. Among the 20 patients in total, 18 were females and two males. Two patients exhibited keloids on bilateral ears, meanwhile eight on the right ear and 10 on the left ear. Twenty-two keloids underwent excision including the two bilateral cases. With regard to the anatomical site, eight were at the ear helix, two at the ear concha, and 12 at the ear lobule. Two patients have undergone keloid excision previously, and they were categorized as secondary cases. The rest 18 patients were primary cases. In the majority of the cases $(n=19)$, the keloids were caused by ear piercing, while in one case, the lesion was caused by a traumatic injury (Table 1 ).

All patients completed the treatment protocol with a followup interval of 6 months. Of the 22 keloid tissues, 20 keloids have been successfully eradicated (90.9\%), while two keloids presented remnant fibrous tissue, showing slight under-correction. The postoperative course related to wound healing was uneventful in all patients. The representative pre- and post-operative images are demonstrated in clinical photographs (Figs. 3, 4).

Table 1. Patient demographics

\begin{tabular}{lc}
\hline Index & Measurement \\
\hline Age $(\mathrm{yr})$ & $22 \pm 5(15-40)$ \\
Sex (female/male) & $18 / 2$ \\
Location & \\
Right/left/bilateral $(\mathrm{n}=20)$ & $8 / 10 / 2$ \\
Helix/concha/lobule $(\mathrm{n}=22)^{a)}$ & $8 / 2 / 12$ \\
Diameter $(\mathrm{cm})$ & $1.7 \pm 0.8(0.9-2.8)$ \\
Follow-up (mo) & $12 \pm 5(6-19)$ \\
Surgical history & \\
Primary/secondary & $20 / 2$ \\
Cause & \\
Piercing/trauma & $21 / 1$ \\
\hline
\end{tabular}

Values are presented as mean \pm standard deviation (range) or number. a)Twenty-two ear keloids from 20 patients have been treated, since two patients presented keloids on bilateral ears. 

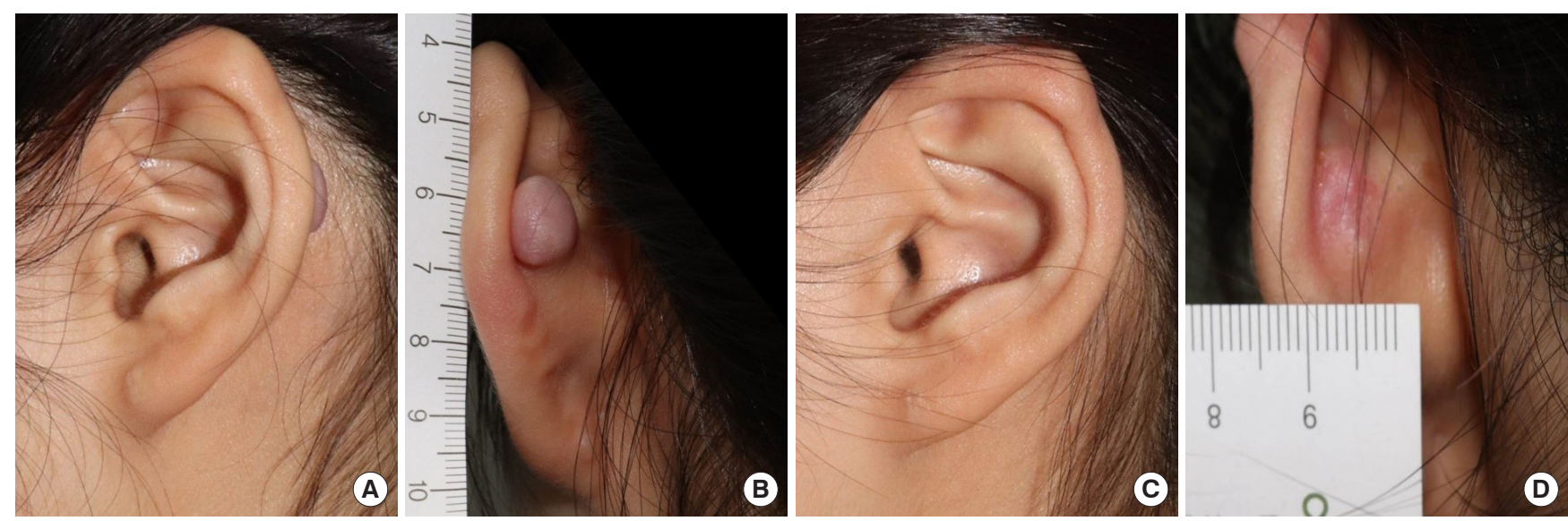

Fig. 3. (A, B) A 20-year-old female patient presented a pedunculated-pattern keloid on the helical rim. The patient underwent surgical excision and adjuvant intermittent pressure therapy. (C, D) The postoperative image at follow-up 8 months showed clinical improvement without complications.
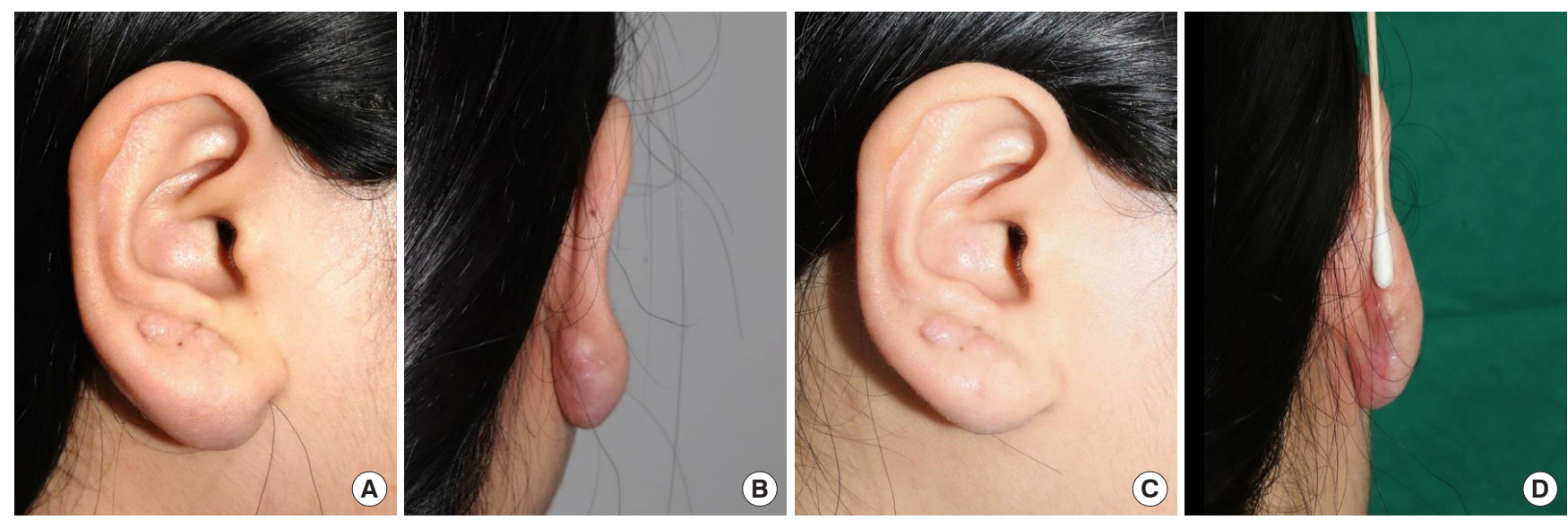

Fig. 4. (A) A 27-year-old female patient presented a protruded keloid on the ear lobule. (B) The patient had undergone surgical excision 1 year before and showed recurrence. We performed additional surgical reduction and adjuvant intermittent pressure therapy. (C, D) The postoperative image at 6 months showed clinical improvement without wound related complications.

The scar assessment scale demonstrated a significant improvement in each index, namely vascularity, pigmentation, thickness, relief, pliability, and surface area (Table 2). With regard to patients' satisfaction in symptoms, 18 patients (20 keloids) were satisfied with the outcome as well as treatment course. Nonetheless, two patients (2 keloids) with remnant keloids showed dissatisfaction on the final outcome. Recurrence of keloid was not presented in overall patients.

Treatment with intermittent magnetic pressure therapy resulted in notable improvement, without any therapy-related discomfort or skin ulceration. During the therapy period, the patients were observed once a month, because pressure-related pain and discomfort could reduce patient compliance. On each visit, pressure maintenance and resting periods were checked, and the patients were encouraged to figure out adequate magnetic strength.
Table 2. Preoperative and postoperative analysis based on scar assessment scale $(n=22)$

\begin{tabular}{lccc}
\hline Index & $\begin{array}{c}\text { Preoperative } \\
\text { measurement }\end{array}$ & $\begin{array}{c}\text { Postoperative } \\
\text { measurement }\end{array}$ & $p$-value \\
\hline Vascularity & $4.5 \pm 1.9$ & $3.2 \pm 1.4$ & $0.02^{\mathrm{a})}$ \\
Pigmentation & $3.8 \pm 1.1$ & $2.4 \pm 0.8$ & $0.01^{\mathrm{a})}$ \\
Thickness & $6.4 \pm 2.3$ & $3.5 \pm 2.1$ & $<0.001^{\mathrm{a})}$ \\
Relief & $3.6 \pm 1.2$ & $2.8 \pm 0.7$ & $0.04^{\mathrm{a}}$ \\
Pliability & $5.7 \pm 1.6$ & $3.0 \pm 1.4$ & $<0.001^{\mathrm{a})}$ \\
Surface area & $6.3 \pm 2.5$ & $3.8 \pm 1.7$ & $0.03^{\mathrm{a}}$ \\
\hline
\end{tabular}

Values are presented as mean \pm standard deviation. a) $p<0.05$.

\section{DISCUSSION}

Pressure therapy combined with surgical excision has been clinically applied worldwide, and the efficacy of this combina- 
tional treatment was well reported in the literature [11]. Compression therapies in keloid and hypertrophic scars have been adopted in various ways, and the related healing mechanisms under controlled pressure generation were demonstrated in several researches. Under compressive circumstances, hypoxia and apoptosis of the keloid tissue occurs with changes of cytokine levels including tumor necrosis factor (TNF)- $\alpha$ or matrix metalloproteinase (MMP)-28 [12,13].

Reno et al. [12] described the regulation of two cytokines involved in scar formation in an in vitro study using postburn hypertrophic scars. In case of continuous elastic compression, interleukin- $1 \beta$ secretion was increased, which was accompanied by dermal apoptosis, whereas TNF- $\alpha$ was down-regulated. Meanwhile, MMP-28, a protease released by keratinocytes in traumatic tissue conditions, was also introduced as a regulator protein for keloid formation related to mechanical compression forces [13]. MMP-28 is a molecular modulator that helps in the restructuring of the skin basal membrane layer. While an environment of mechanical tissue compression leads to MMP-28 increment in normotrophic scars, decreased expressions of the protein in the same condition were observed in hypertrophic scars. This means that mechanical compression forces may affect the level of release and expression of MMP-28; therefore, the compressive environment modulates the wound remodeling phase in hypertrophic scars.

These compression-related molecular-biological modulators regulate the physiological pathways of cellular apoptosis, which are genetically programmed for maintaining tissue integrity and homeostasis, and microscopically marked as nuclear condensation with DNA degradation $[14,15]$. In a comparative study using hypertrophic scar and normal skin, apoptotic cells with degraded DNA were observed more frequently on the normal skin dermis, while fibroblasts derived from hypertrophic scars were less prone to apoptotic triggering [16,17].

With regard to the magnetic pressure application protocol, we hypothesized that intermittent application could be more efficient than continuous usage in tissue remodeling. This concept was based on the result of an animal study by Shoman et al. [18], in which the applications of intermittent forces with adequate loading and unloading intervals helped maintain the physiologic conditions of capillary structures. Without proper unloading intervals, the chances of unpredictable bleeding and vascular destruction or disappearance were increased.

In a study on intermittent negative pressure effect on the macro- and microcirculation of lower extremities, intermittent application induced better blood flow than the constant protocol [19]. Intermittent negative pressure resulted in higher blood flow velocity, skin blood flow, and skin temperature. Mean- while, the constant negative pressure group showed decreased blood flow velocity, skin blood flow, and skin temperature. Accordingly, increased foot perfusion was observed after the application of intermittent negative pressure.

We broke one application cycle into three separated sub-cycles, rather than applying the device for 9 hours continuously. Each sub-cycle included 3 hours of pressure-on period followed by 2 hours of pressure-off period, in which the devices were removed and accordingly, the pressures were relieved. When the third pressure-on period finished, the cycle of the day which composed of total 15 hours of intermittent on- and off-magnetic pressure was finalized. Our protocol has been designated to avoid keloid recurrence as well as pressure-related complication. The unwanted sequels are generated, when application method causes discomfort and aversion. In this context, we designated the 3-hour loading and 2-hour unloading protocol, which included a long relieving period relatively, to achieve efficient application and patients' comfort during regular daily activities.

Two of the patients in the current study exhibited remnant keloid tissue $(n=2,9.1 \%)$, indicating slight under-correction. Various surgical excision protocols have been used to reduce keloid tissues, with complete excision without any remaining overlying skin, namely a keloid marginal flap, being one of them [20]. Eradication is advantageous in reducing the keloid burden; however, surgeons should be cautious to avoid soft tissue tension in the post-excision state. Excision is another surgical method to treat keloids [21]. This technique utilizes the overlying epidermis and the thin dermis at the keloid marginal areas; therefore, the soft skin layer can be used for wound closure, avoiding related complications. We employed the excision method to facilitate the wound healing process. Nonetheless, sufficient resection of the keloid burden is a surgical goal, and additional reduction should be considered when remnant fibrous tissue is noted during the intraoperative wound examination. In this context, surgeons should evaluate the keloid margin cautiously in both preoperative and intraoperative procedures.

This study has a few limitations. The sample size was relatively small and there was no control group. Additionally, considering its retrospective nature and relatively short period of postoperative follow-up period, studies with longer follow-up and largersized patient samples are needed to confirm the long-term efficacy of our management protocol.

In conclusion, the intermittent pressure therapy as per our post-excision protocol can lead to efficient postoperative management, avoiding the recurrence of ear keloids. We laid more emphasis on the maintenance of the natural anatomical posi- 
tion of innate soft tissues rather than on the pressure effect. A thorough understanding of the pathomechanisms causing keloid formation can help identify pathways that serve as adequate targets to achieve an effective therapy for this intractable disorder.

\section{NOTES}

\section{Conflict of interest}

No potential conflict of interest relevant to this article was reported.

\section{Ethical approval}

The study was approved by the Institutional Review Board of Konkuk University School of Medicine (IRB No. KUMC 201906-009) and performed in accordance with the principles of the Declaration of Helsinki. Written informed consents were obtained.

\section{Patient consent}

The patients provided written informed consent for the publication and the use of their images.

\section{ORCID}

Dongkeun Jun ～https://orcid.org/0000-0001-9017-9929

Donghyeok Shin https://orcid.org/0000-0002-8450-4411

Hyungon Choi https://orcid.org/0000-0002-3816-1286

Myungchul Lee https://orcid.org/0000-0002-9721-0092

\section{REFERENCES}

1. Unahabhokha T, Sucontphunt A, Nimmannit U, Chanvorachote $\mathrm{P}$, Yongsanguanchai $\mathrm{N}$, Pongrakhananon V. Molecular signalings in keloid disease and current therapeutic approaches from natural based compounds. Pharm Biol 2015;53:457-63.

2. Andrews JP, Marttala J, Macarak E, Rosenbloom J, Uitto J. Keloids: the paradigm of skin fibrosis-pathomechanisms and treatment. Matrix Biol 2016;51:37-46.

3. Jones ME, McLane J, Adenegan R, Lee J, Ganzer CA. Advancing keloid treatment: a novel multimodal approach to ear keloids. Dermatol Surg 2017;43:1164-9.

4. Akoz T, Gideroglu K, Akan M. Combination of differenchniques for the treatment of earlobe keloids. Aesthetic Plast Surg 2002;26:184-8.

5. Park TH, Seo SW, Kim JK, Chang CH. Outcomes of surgical excision with pressure therapy using magnets and identification of risk factors for recurrent keloids. Plast Reconstr Surg 2011;128:431-9.
6. Tanaydin V, Beugels J, Piatkowski A, Colla C, van den Kerckhove E, Hugenholtz GC, et al. Efficacy of custom-made pressure clips for ear keloid treatment after surgical excision. J Plast Reconstr Aesthet Surg 2016;69:115-21.

7. Daya M, Nair V. Traction-assisted dermatogenesis by serial intermittent skin tape application. Plast Reconstr Surg 2008;122: 1047-54.

8. Shim HS, Choi JS, Kim SW. A role for postoperative negative pressure wound therapy in multitissue hand injuries. Biomed Res Int 2018;2018:3629643.

9. Chowdhry SA, Wilhelmi BJ. Comparing negative pressure wound therapy with instillation and conventional dressings for sternal wound reconstructions. Plast Reconstr Surg Glob Open 2019;7:e2087.

10. Park TH, Rah DK. Successful eradication of helical rim keloids with surgical excision followed by pressure therapy using a combination of magnets and silicone gel sheeting. Int Wound J 2017;14:302-6.

11. Chang CH, Song JY, Park JH, Seo SW. The efficacy of magnetic disks for the treatment of earlobe hypertrophic scar. Ann Plast Surg 2005;54:566-9.

12. Reno F, Sabbatini M, Lombardi F, Stella M, Pezzuto C, Magliacani $\mathrm{G}$, et al. In vitro mechanical compression induces apoptosis and regulates cytokines release in hypertrophic scars. Wound Repair Regen 2003;11:331-6.

13. Reno F, Sabbatini M, Stella M, Magliacani G, Cannas M. Effect of in vitro mechanical compression on Epilysin (matrix metalloproteinase-28) expression in hypertrophic scars. Wound Repair Regen 2005;13:255-61.

14. Thompson CB. Apoptosis in the pathogenesis and treatment of disease. Science 1995;267:1456-62.

15. Kawamoto Y, Nakajima YI, Kuranaga E. Apoptosis in Cellular Society: communication between apoptotic cells and their neighbors. Int J Mol Sci 2016;17:E2144.

16. Wassermann RJ, Polo M, Smith P, Wang X, Ko F, Robson MC. Differential production of apoptosis-modulating proteins in patients with hypertrophic burn scar. J Surg Res 1998;75:74-80.

17. Chodon T, Sugihara T, Igawa HH, Funayama E, Furukawa $H$. Keloid-derived fibroblasts are refractory to Fas-mediated apoptosis and neutralization ofautocrine transforming growth factor-betal can abrogate this resistance. Am J Pathol 2000;157: 1661-9.

18. Shoman K, Sato Y, Nishikawa E, Kudo Y, Yamamoto T, Iida J. Evaluation of the effectiveness of intermittent mechanical pressure with short loading duration: new type of intermittent force for orthodontic treatment. Eur J Orthod 2017;39:264-9.

19. Sundby OH, Hoiseth LO, Mathiesen I, Jorgensen JJ, WeedonFekjer H, Hisdal J. Application of intermittent negative pressure 
on the lower extremity and its effect on macro- and microcirculation in the foot of healthy volunteers. Physiol Rep 2016; 4:e12911.

20. Stahl S, Barnea Y, Weiss J, Amir A, Zaretski A, Leshem D, et al. Treatment of earlobe keloids by extralesional excision com- bined with preoperative and postoperative "sandwich" radiotherapy. Plast Reconstr Surg 2010;125:135-41.

21. Lee Y, Minn KW, Baek RM, Hong JJ. A new surgical treatment of keloid: keloid core excision. Ann Plast Surg 2001;46:135-40. 